\title{
Aggregation of nanoscale iron oxyhydroxides and corresponding effects on metal uptake, retention, and speciation: I. Ionic-strength and pH
}

\author{
Dale, J.G., Stegemeier, J.P., and Kim, C.S.*
}

Revised submission 8/20/14

School of Earth and Environmental Sciences, Schmid College of Science \& Technology, Chapman University, Orange, CA 92866

*contact email: cskim@ chapman.edu 


\section{ABSTRACT}

The capacity of nanosized particles to adsorb and sequester dissolved metals can be significantly impacted by the mechanism and extent of aggregation the particles have undergone, which in turn can affect the long-term fate and transport of potentially toxic metals in natural aqueous systems. Suspensions of monodisperse nanoscale iron oxyhydroxides were synthesized and subjected to increased $\mathrm{pH}(\mathrm{pH} 8.0,10.0)$ or ionic strength $\left(0.1 \mathrm{M}, 1.0 \mathrm{M} \mathrm{NaNO}_{3}\right)$ conditions to induce various states of aggregation prior to conducting macroscopic adsorption/desorption experiments with dissolved $\mathrm{Cu}(\mathrm{II})$ or $\mathrm{Zn}(\mathrm{II})$. The metal adsorption and retention capacities of the nanoparticle aggregates were compared to one another and to non-aggregated control nanoparticles, while the mode(s) of metal sorption to the nanoparticle surfaces were characterized by extended X-ray absorption fine structure (EXAFS) spectroscopy analysis.

With increasing aggregation by both $\mathrm{pH}$ and ionic strength, the proportion of introduced zinc adsorbed to the iron oxyhydroxide nanoparticles progressively decreased from $45 \%$ on the monodispersed control particles to as low as $16 \%$ on the aggregates, while the proportion of introduced zinc retained upon desorption (obtained by lowering the suspension $\mathrm{pH}$ ) increased from $7 \%$ on the control particles to as much as $17 \%$ on the aggregated particles. Copper exhibited a subtler trend of only slightly declining uptake (from $43 \%$ to $36 \%$ ) and retention (from 35\% to 30\%) with increasing aggregation state. EXAFS analysis was consistent with the macroscopic results, showing relatively little change in $\mathrm{Cu}$ speciation between samples analyzed before and after the desorption step but significant increases in $\mathrm{Zn}-\mathrm{Fe}$ interatomic distances and coordination numbers after desorption. This suggests the presence of both strongly- and weakly-bound zinc ions; the latter are likely affiliated with less stable, more distorted surface sorption sites and are thus more readily desorbed, resulting in the retention of zinc that is bound to more stable, less-distorted sorption sites. For both metals, inner- 
sphere bidentate sorption appears to dominate the sorption process to the nanoparticle aggregates, with potential structural incorporation into the aggregates themselves.

\section{INTRODUCTION}

Metal contamination is a serious concern for both human and environmental health. Even common metals such as zinc and copper, which are required dietary components, can cause negative health effects when they reach acute or chronic toxicity levels, disrupting ecosystems and causing sickness in humans. Such elevated levels can be found near anthropogenic sources of metal contamination including mining sites, which often feature low $\mathrm{pH}$ waters and high dissolved metal concentrations as a result of acid mine drainage (AMD) (BLOWES et al., 2003; NoRDSTROM and ALPERS, 1999). The onset of rainfall events following a prolonged dry season in these areas often produces a "first flush" of surface water runoff, where periodic pulses of acidic water result in elevated aqueous metal concentrations through sediment transport/deposition, mineral dissolution and metal desorption processes, as has been documented in locations such as Iron Mountain, CA (DRUSCHEL et al., 2004; JAMIESON et al., 2005; NORDSTROM et al., 2000). The abundance of similarly contaminated mine sites throughout the world and the subsequent threat they pose to the health of surrounding inhabitants and ecosystems makes the effective remediation of these areas a high priority.

The correlation between the adsorption of dissolved metals to mineral particle surfaces and the corresponding long-term fate of such metals in natural aqueous systems is generally well known. The mineral-water interface can control the mobility and potential bioavailability of dissolved metals through adsorption processes which may lead over time to (co-)precipitation, surface precipitation, and/or structural incorporation into the solid phase, facilitating the sequestration of metals in sediments

(FORD et al., 2001; GRAFE et al., 2004; HERBERT JR, 1996). Although the adsorption of dissolved metal species to minerals has been the focus of numerous studies, the tendency of metals to desorb and return 
to aqueous solution has been relatively less well assessed despite the fundamental importance of desorption processes in the long-term availability of metals in natural systems, particularly those that may undergo geochemical (e.g. pH, ionic strength) changes over time. Past studies have also established that most minerals have non-homogeneous surfaces involving multiple surface binding sites and strengths (BENJAMIN and LECKIE, 1981; HIEMSTRA and VAN RIEMSDIJK, 1996; VILLALOBOS et al., 2009), which has implications for the retention and sequestration of sorbed metals under varying geochemical conditions.

Iron oxyhydroxides are considered to be the dominant reactive mineral phase in marine and lake sediments (VAN DER ZEE and ROBERTS, 2003) due to their relative ubiquity, high surface area, and strong capacity for metal sorption (DYER et al., 2004; XU et al., 2006); such phases are particularly abundant in AMD environments, which are known for a characteristic red staining along riverbeds from freshly-precipitated iron hydroxides. Furthermore, iron oxyhydroxide phases have been observed in natural systems at the nanoscale (PENN et al., 2001; VAN DER ZEE and ROBERTS, 2003), likely contributing to their high reactivity with dissolved metals. However, under most natural aqueous conditions, nanoparticulate iron oxyhydroxides will rapidly aggregate following formation (BANFIELD et al., 2000; GILBERT et al., 2007; YUWONO et al., 2012). Despite the often repeated possibility of using nanoparticulate iron oxyhydroxides and other minerals as sorbents for dissolved metals, few studies have focused on the effects of aggregation on their sorption properties.

The aggregation of nanoparticles is likely to impact their adsorption and retention capacities in a variety of potential ways, including reducing available surface areas (leading to lowered adsorption) and aiding the structural incorporation of metals into nanoporous regions (decreasing desorption and increasing retention) (KIM et al., 2008). Additionally, the mechanism of aggregation, such as $\mathrm{pH}$ (GILBERT et al., 2007), ionic strength (LI and XU, 2008), and temperature (GILBERT et al., 2009) is known to impact the formation of the resulting aggregates. These morphological variations caused by 
aggregation conditions may affect the sorption of metals, playing an important role in the binding strength of those metals and therefore their long-term stability in the resulting solid phases. Structural models for the complex interactions which may arise from sorption onto/into aggregates do not currently exist in the literature.

The purpose of this study is to examine the effects of different aggregation mechanisms on the adsorption and desorption behavior of metals onto/from iron oxyhydroxide nanoparticles, with the initial hypothesis that aggregation reduces adsorption but enhances the retention (i.e. reduces the desorption) of metals that are sorbed to nanoparticle aggregates. This involves a combination of macroscopic batch adsorption/desorption experiments conducted to quantify metal uptake and retention and extended X-ray absorption fine structure (EXAFS) spectroscopy to probe the local structural environment of the sorbed metals and determine the mode(s) of metal binding. Such structural information can then be used to 1) generate models for the uptake and retention of dissolved metals to nanoparticles and their aggregates; 2) explain the strength of metal binding to iron oxyhydroxide nanoparticles under a range of environmental conditions; and 3) assess the overall effectiveness of aggregated iron oxyhydroxide nanoparticles at metal retention in aqueous systems. Nanoparticle aggregation by increases in $\mathrm{pH}$ and ionic strength are explored in this, the first of a two-part study, with the second part focusing on aggregation as a function of temperature and time.

\section{METHODS}

\subsection{Iron oxyhydroxide nanoparticle synthesis}

A stock suspension of iron oxyhydroxide nanoparticles was created from reagent grade materials using a microwave flash synthesis technique (GuYODO et al., 2003). A $0.25 \mathrm{M} \mathrm{NaHCO}_{3}$ solution was filtered through a $0.20 \mu \mathrm{m}$ Nylon filter and added dropwise to an equal volume of $0.20 \mathrm{M}$ $\mathrm{Fe}\left(\mathrm{NO}_{3}\right)_{3}$ under constant agitation using a stir bar. The mixture was prepared in an HDPE bottle, 
sealed, and shaken on a rotator table for three hours, with the solutions vented to release generated $\mathrm{CO}_{2}$ at 30-minute intervals. The nucleation of iron oxyhydroxide nanoparticles was then initiated by heating the solution in a conventional microwave on high power at 40-second intervals with $2-3$ seconds of manual stirring in between heating intervals. As soon as boiling was attained, the bottle was immediately immersed in an ice bath for rapid cooling in order to halt the growth of the nanoparticles.

Once returned to room temperature, the nanoparticle suspension was transferred to 1000 MWCO dialysis tubing and placed in a large volume of DI water which was replaced a minimum of nine times over the course of three days, monitoring $\mathrm{pH}$ and conductivity until both approached those of control solution conditions ( $\mathrm{pH} 5.0$ and $\left.0.001 \mathrm{M} \mathrm{NaNO}_{3}\right)$. These conditions were selected for the control solution because they were empirically found to prevent substantial aggregation over long periods of time (i.e. weeks-months). The synthesized nanoparticles in their dialysis tubing were then placed into a prepared control solution for another three days with a minimum of nine solution replacements until no significant change in $\mathrm{pH}$ or conductivity could be detected. Once equilibrium was reached, the iron oxyhydroxide nanoparticle suspension was transferred to 1L HDPE bottles and stored in a $4^{\circ} \mathrm{C}$ refrigerator. Nanoparticles were used for experiments within 2 days of completed synthesis.

\subsection{Nanoparticle aggregation}

Aqueous conditions were selected to induce aggregation of the control nanoparticle suspensions at levels that were anticipated to lead to differences in metal uptake and retention. Increasing ionic strength causes aggregation by providing sufficient counter-ions to reduce the thickness of the electrical double layer, allowing the nanoparticles to position themselves closer together, and enabling aggregation to proceed (HoU et al., 2007; LI and XU, 2008). Alternatively, solution pH influences surface charge density, and as the $\mathrm{pH}$ approaches the point of zero charge $\left(\mathrm{pH}_{\mathrm{pzc}}\right.$, which for Fe- 
hydroxides ranges from 7-9 (DZOMBAK and MOREL, 1990)), the electrostatic repulsion between particles is reduced (GILBERT et al., 2009; Stumm and Morgan, 1996). As the net surface charge density approaches zero, particles are able to position themselves in close proximity to each other, leading to aggregation (LOWRY et al., 2004). Adsorption, desorption, dissolution and precipitation reactions have all been associated with the surface charge and electrical double layer of minerals (STUMM, 1997).

Aggregation-inducing solutions were prepared using deionized water, reagent grade $\mathrm{NaNO}_{3}$, and $0.1 \mathrm{M} \mathrm{NaOH}$ or $\mathrm{HNO}_{3}$ generated from $70 \%$ or $50 \%$ stock solutions, respectively. The parameters for the aggregation-inducing solutions are summarized in Table 1. To induce aggregation, $25 \mathrm{~mL}$ aliquots of the control nanoparticle suspension were poured into separate sections of $1000 \mathrm{MWCO}$ dialysis tubing and then placed into 1 L HDPE bottles filled with their respective aggregation solution. The suspensions remained in the aggregation solutions for five days, during which time the solutions were replaced a minimum of three times per day. Visual inspection of the suspensions confirmed that the degree of aggregation increased progressively with both $\mathrm{pH}$ and ionic strength; this was characterized further with dynamic light scattering measurements as described later.

Conductivity and $\mathrm{pH}$ measurements were recorded prior to each solution replacement to ensure equilibrium was reached. After aggregation, the samples were returned to the control solution ( $\mathrm{pH}$ 5.0, $0.001 \mathrm{M} \mathrm{NaNO}_{3}$ ) and allowed to equilibrate for another three days with a minimum of three solution replacements per day while the $\mathrm{pH}$ and conductivity were monitored. This re-equilibration was performed in order to bring the different nanoparticle aggregate suspensions back to the same initial control solution conditions before beginning the subsequent adsorption/desorption experiments. Even after re-equilibration, samples where either $\mathrm{pH}$ or ionic strength had been increased showed both visible and variable degrees of aggregation and settling relative to the control sample, which remained a homogeneous suspension of particles with no visible settling. This suggests that the extent of 
aggregation by these methods, even after re-equilibration under control conditions, is largely irreversible.

\subsection{Characterization of nanoparticle aggregates}

Characterization of the iron oxyhydroxide nanoparticle aggregates was conducted in order to ensure consistency between batches and to assess the varying degrees of aggregation between samples and within a given sample throughout the macroscopic uptake/desorption experiments. A solids concentration of $6.4 \pm 0.2 \mathrm{~g} / \mathrm{L}$ was determined gravimetrically by drying measured volumes of suspension in drying dishes. Gilbert et al. previously determined the $\mathrm{pH}$ of zero net surface charge $\left(\mathrm{pH}_{\mathrm{znsc}}\right)$ of similarly prepared material to be 8.6 by potentiometric titrations (GILBERT et al., 2007). A FEI/Philips CM-20 conventional transmission electron microscope (TEM) was used to determine nanoparticle size and shape as well as to assess the qualitative extent of aggregation among the samples. The surface areas of air-dried and ground aliquots of the control sample and the various aggregated samples were determined using a Beckman-Coulter SA3100 BET surface area analyzer. All solids concentrations and surface areas were measured in triplicate.

Powder X-ray diffraction patterns were collected on the control iron oxyhydroxide nanoparticles as well as those aggregated at $\mathrm{pH} 8.0$ and 10.0 and at ionic strength 0.1 and $1.0 \mathrm{M} \mathrm{NaNO}_{3}$ in order to determine mineral phase composition and the degree of crystallinity of the samples. X-ray diffraction was conducted at beamline 11-3 of the Stanford Synchrotron Radiation Lightsource (SSRL) at an energy of $12735 \mathrm{eV}(0.9735 \AA$ A $)$ calibrated using a Si(311) crystal monochromator. Exposures were collected for 90 seconds on a Mar345 CCD detector (except the $\mathrm{pH} 8.0$ sample which was collected for 30 seconds and scaled accordingly for comparison), integrated using the software program fit2D, and background-subtracted using the software program XRD-BS. Samples were air-dried in dishes and ground with a mortar and pestle before analysis. 
Dynamic light scattering (DLS) analysis was conducted on the control nanoparticles and resulting aggregates using a Beckman Coulter Delsa Nano C dynamic light scattering system. Samples were diluted 10x in DI water for optimal particle detection by DLS, mixed thoroughly in their cuvettes using a $1000 \mu \mathrm{L}$ autopipet just before analysis, and then analyzed with a $50 \mu \mathrm{m}$ laser aperture size to determine average hydrodynamic diameter and polydispersity. For strongly aggregated samples, the settled fraction of the sample was collected for dilution. Each sample was analyzed under the following conditions designed to mimic those of the adsorption/desorption experiments described in the next section: 1) after re-equilibration in control solution for a period of 3 days; 2) after $\mathrm{pH}$ adjustment using 0.1 $\mathrm{M} \mathrm{NaOH}$ to a value of 7.0 for a period of 24 hours; and 3) after adjustment of the prior suspension back to $\mathrm{pH} 5.0$ using $0.1 \mathrm{M} \mathrm{HNO}_{3}$ for approximately 2 hours. Each sample was analyzed between 2-6 times with 70 cumulative measurements collected per trial. This allowed a reliable index to characterize the average hydrodynamic radius and degree of polydispersity of the particles/aggregates in each nanoparticle suspension at various stages of the aggregation, adsorption, and desorption processes to which they were subjected, and thus to confirm quantitative differences in aggregation state between the different suspensions throughout the course of the experiment.

The morphologies of these synthesized and aggregated nanoparticles were previously examined using in situ small-angle X-ray scattering (SAXS) (GILBERT et al., 2009). Analysis of the SAXS patterns indicates that the control samples persist as largely unaggregated nanoparticles due to mutual repulsive interactions between particles at $\mathrm{pH} 5$, while increases in $\mathrm{pH}$ and ionic strength lead to increasingly larger fractal nanoparticle aggregates of loosely-attached nanoparticle clusters containing substantial volumes of interstitial water. Importantly, structural models generated and comparison of their simulated SAXS patterns with experimental SAXS data concluded that the $\mathrm{pH}$ - and ionic strengthaggregated nanoparticles adopt very open aggregate structures that are unlikely to substantially reduce the reactive surface area accessible to aqueous ions. These aggregates have been shown to be stable in 
the absence of physical stresses for up to 3 months, suggesting that the sorption and desorption conditions were likely to have little overall effect on the aggregates, preserving their different aggregation states over time and with respect to one another (GILBERT et al., 2007).

\subsection{Metal adsorption/desorption studies with nanoparticle aggregates}

Metal uptake to the control and aggregated nanoparticles was initiated by mixing $100 \mathrm{~mL}$ of either a $5 \mathrm{mM} \mathrm{Cu}\left(\mathrm{NO}_{3}\right)_{2}$ or $\mathrm{Zn}\left(\mathrm{NO}_{3}\right)_{2}$ solution with $25 \mathrm{~mL}$ of nanoparticle suspension which had been diluted with deionized water to a volume of $900 \mathrm{~mL}$, obtaining a final volume of $1.00 \mathrm{~L}$, solids concentration of $0.16 \mathrm{~g} / \mathrm{L}$, and $\mathrm{Cu}(\mathrm{II})$ or $\mathrm{Zn}(\mathrm{II})$ concentration of $0.5 \mathrm{mM}$ while maintaining $\mathrm{pH}$ and ionic strength at control conditions. Based on calculations using the software package Geochemist's Workbench® v7.0 (BETHKE, 1996; BETHKE, 2002), these concentrations were determined to be below the saturation indices for any potential $\mathrm{Cu}$ - or $\mathrm{Zn}$-precipitates to form within the $\mathrm{pH}$ range used in these experiments.

The $\mathrm{pH}$ of the suspension was then increased to $6.0 \pm 0.1$ or $7.0 \pm 0.1$ using $20 \mu$ aliquots of 1.0 $\mathrm{M} \mathrm{NaOH}$ to maximize uptake of $\mathrm{Cu}(\mathrm{II})$ or $\mathrm{Zn}(\mathrm{II})$, respectively. These $\mathrm{pH}$ levels were determined by pH-based macroscopic uptake experiments previously conducted on monodispersed iron oxyhydroxide nanoparticles prepared using the same synthesis method and confirmed by calculations and measurements made by Dzombak and Morel (1990). The suspensions were capped and stirred with Teflon-coated magnetic stir bars at room temperature for 24 hours to allow the adsorption process to reach equilibrium before being split into two aliquots, the first of which is hereafter referred to as the

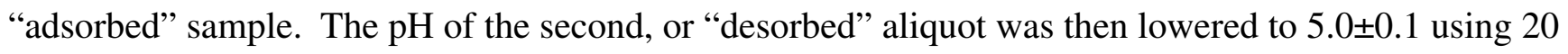
$\mu$ aliquots of $1.0 \mathrm{M} \mathrm{HNO}_{3}$ and stirred for 2 hours. Desorption trials conducted for shorter periods of time showed no substantial change in desorption after 30 minutes. Experimental errors for the 
macroscopic work were established by running triplicate adsorption and desorption experiments with the control sample. The experimental errors for zinc uptake and retention were measured to be $1.4 \%$ and $1.1 \%$ respectively, while those for copper were $0.8 \%$ and $2.2 \%$ respectively.

After both the adsorption and desorption steps, the sample suspensions were centrifuged for 15 minutes at $3000 \mathrm{RPM}$ in $50 \mathrm{~mL}$ Falcon tubes. The resulting supernatants were decanted and filtered through $0.20 \mu \mathrm{m}$ Nylon filters, then acidified to $\mathrm{pH} \leq 2.0$ by adding $70 \% \mathrm{HNO}_{3}$ in $20 \mu \mathrm{L}$ aliquots prior to $\mathrm{Cu}, \mathrm{Zn}$, and/or Fe analysis using a Thermo Solaar S4 Atomic Absorption Spectrometer. Iron analysis of the supernatants revealed insignificant concentrations at or below detection limits, suggesting that nanoparticle dissolution was minimal during the experiment. The remaining solid pellets were collected and the excess water was wicked away using Whatman filter paper before loading as damp pastes into 1.5 mm-thick Teflon sample holders and sealing with Kapton tape for EXAFS analysis.

\subsection{Extended X-ray absorption fine structure (EXAFS) spectroscopic analysis}

EXAFS spectroscopy of selected samples was performed on beamlines 10-2 and 11-2 at SSRL. Data was collected with a $\operatorname{Si}(220) \phi=90^{\circ}$ monochromator crystal detuned up $20 \%$ or $40 \%$ on beamlines 10-2 and 11-2, respectively, from the maximum beam intensity in order to reject higher order harmonic signals. Zn K-edge $(9659 \mathrm{eV})$ and $\mathrm{Cu}$ K-edge $(8979 \mathrm{eV})$ spectra were collected on the prepared wet pastes at room temperature in fluorescence yield mode using a 13 (BL 10-2) or 32 (BL 11-2) element high-throughput germanium detector. This technique is advantageous compared to the use of transmission- or ion-chamber-collected fluorescence because of its decreased noise and increased signal for low concentration samples (STERN and HEALD, 1979; WAYCHUNAS and BROWN, 1994). Soller slits and aluminum filters were used to minimize elastic scattering and interference from the Fe 
K-edge fluorescence, respectively. Zinc and copper metal foils were used as monochromator energy calibrants.

Collected spectra were analyzed using the SIXPack software package version 0.64 (WEBB, 2005). Scans were deadtime-corrected for potential loss of signal due to finite photon detection times and then averaged together. After background subtraction, the data were converted to $\mathrm{k}$-space with a $\mathrm{k}^{3}$ weighting ( $\mathrm{E}_{0}$ values were set at values $20 \mathrm{eV}$ above the edge for each metal) and Fourier transformed. The chi files were fit over a k-range of 3.0-13.1 $\AA^{-1}$ for $\mathrm{Cu}$ and 3-11.8 $\AA^{-1}$ for $\mathrm{Zn}$. All samples were fit with phase and amplitude functions from model single-shell scattering paths generated in SIXPack using Feff61 (REHR et al., 1991).

\section{RESULTS}

\subsection{Nanoparticle characterization}

\subsubsection{X-ray diffraction}

The X-ray diffraction patterns of the control (unaggregated) iron oxyhydroxide nanoparticles and nanoparticle samples aggregated at $0.1 \mathrm{M}$ ionic strength, $1.0 \mathrm{M}$ ionic strength, $\mathrm{pH} 8$, and $\mathrm{pH} 10$ are presented in Figure 1. The XRD patterns of the aggregates appear generally similar to that of the control suspension, suggesting that the aggregation process does not substantially alter the phase or degree of crystallinity of the nanoparticles. However, the aggregate generated at $\mathrm{pH} 10$ exhibits greater intensity and more resolved peaks than the other samples, consistent with substantially larger particle sizes. Comparison with PDF standards of 6-line ferrihydrite and goethite indicate that the XRD patterns of all nanoparticle suspensions match both standards but are primarily consistent with 6-line ferrihydrite, although some subtle peaks unique to goethite are also present. This is consistent with the earlier identification of ferrihydrite nanoparticles through this synthesis method, although the oriented 
aggregation of these nanoparticles with heating/aging has been demonstrated to yield acicular goethite nanorods (BURLESON and PENN, 2006; PENN et al., 2006).

\subsubsection{Transmission electron microscopy}

Representative TEM images of the control iron oxyhydroxide nanoparticles as well as those aggregated by ionic strength $(1.0 \mathrm{M})$ and $\mathrm{pH}(10.0)$ reveal that the control nanoparticles are generally well dispersed, with a relatively minor degree of aggregation visible that may have occurred during the drying of the particles on the TEM sample grid (Figure 2b). In contrast, the two aggregated samples

(Figure 2a, 2c) show the formation of denser, more complex multi-particle aggregates. These aggregates are generally disordered but occasionally exhibit signs of oriented aggregation as reported in prior studies of nanogoethite (GUYODO et al., 2003; PENN, 2004). Specifically, parallel lattice fringes can be seen across aggregated particles, although electron diffraction only yields a singular peak which could not be used to conclusively for phase identification. In all samples, the individual particles appear to be spheroidal in shape and approximately 3-4 $\mathrm{nm}$ in diameter. Assuming the particles are spherical with an average diameter of $3.5 \mathrm{~nm}$ as determined by TEM analysis of several hundred particles by Guyodo et al. (GUYODO et al., 2003) and $4.27 \mathrm{~g} / \mathrm{cm}^{3}$ for the density of goethite ( $\left.\alpha \mathrm{FeOOH}\right)$ (SCHWERTMANN and CORNELL, 2000), the geometric surface area of the control sample was approximated to be $401 \mathrm{~m}^{2} / \mathrm{g}$.

\subsubsection{Surface area analysis}

The BET surface area measurements of the control sample and the various aggregates are listed in Table 1. The surface area trends run somewhat counter to expectations, with the $\mathrm{pH}$-aggregated samples returning progressively higher surface areas than the dispersed control sample. It is important, 
however, to note that BET surface area analysis requires complete drying of the nanoparticle suspensions and then grinding the resulting dried solids, both of which likely alter the reactive surface area of the particles/aggregates in suspension. Therefore, aggregation induced as an artifact of the drying process may result in a greater loss of surface area in the control sample than in the other (already) aggregated samples, offering an explanation for the trend reported. The fact that all measured surface areas are between $10-25 \%$ less than the geometrically calculated surface area of the dispersed nanoparticles supports the assumption that the variable loss of surface area is due to the drying process that all samples undergo prior to BET analysis.

Difficulties in accurately measuring the reactive surface areas of nanoparticles formed and aggregated under aqueous conditions have been noted by others as well. Cwiertny et al. (2008) report values of BET measured surface areas for goethite nanoparticles that are approximately $50 \%$ of their estimated TEM surface areas and therefore should not be relied upon to assess differences in surface site density or reactivity in suspension. The study concludes that the reactive surface areas of aggregated particles in suspension are unknown and that differences in nanoparticle reactivity as a function of aggregation may be due to intrinsic reactivity, site densities, or surface area. Madden and Hochella (2005) also noted that BET surface area data for hematite nanoparticles less than $7.5 \mathrm{~nm}$ in length appear to be highly inaccurate and instead calculated surface areas geometrically using a combination of TEM and AFM data. In light of these uncertainties, subsequent macroscopic results are presented without surface area normalization in order to avoid introducing potential artifacts into the results. The relatively loose degree of aggregation induced by changes in $\mathrm{pH}$ and ionic strength, in which water remains present throughout the aggregation process and likely within the interparticle pore spaces formed during aggregation, suggests that changes in reactive surface area through aggregation by increased $\mathrm{pH}$ and ionic strength are likely to be less dramatic than when aggregation is induced by drying, freezing, or heating (GILBERT et al., 2009). However, without accurate surface area data of the 
particles/aggregates in suspension, our conclusions are necessarily based on the assumption that the macroscopic adsorption and desorption trends observed outweigh potential changes in surface area as a function of aggregation method.

\subsubsection{Dynamic light scattering}

Average particle/aggregate hydrodynamic diameters, polydispersity indices, and associated standard deviations for the control and aggregated samples under the various conditions of the macroscopic adsorption/desorption experiments are displayed in Table 2. The results show that after re-equilibration in the control solution for 3 days, the samples maintain significantly distinct aggregation states, indicating that redispersion or disaggregation is not sufficient during reequilibration to fully reverse the aggregation process. Increases in $\mathrm{pH}$ and ionic strength generally correlate with increasing average diameters and polydispersity indices, consistent with earlier SAXS analyses and the visual appearances of the suspensions. The ability to conduct DLS measurements of aqueous suspensions to assess aggregation is thus a clear advantage over BET surface area analysis, which requires initial drying of suspensions.

Upon increasing the solution $\mathrm{pH}$ from the re-equilibration value of 5.0 to 7.0 for a period of 24 hours (thus simulating the adsorption step of the experiment), the average hydrodynamic diameters of the control and the $\mathrm{pH} 8$ aggregates increase while the other aggregate suspensions remain relatively comparable; however, the relative size differences between samples from the re-equilibration conditions are still preserved. Similarly, after lowering the $\mathrm{pH}$ back to 5.0 for a period of 2 hours to simulate the desorption step of the experiment, the average diameters and polydispersities generally decrease yet the relative differences between suspensions are still retained. This suggests that macroscopic differences in $\mathrm{Zn}$ or $\mathrm{Cu}$ adsorption to and desorption from the aggregates can be largely ascribed to the samples' relative differences in aggregation state and not exclusively to changes in 
aggregation state resulting from the $\mathrm{pH}$ changes required to conduct the metal adsorption/desorption experiments.

\subsection{Zinc sorption}

\subsubsection{Macroscopic results}

The macroscopic zinc sorption data in Figure 3 shows the percentage of zinc adsorbed following the uptake step and retained following the desorption step onto the different nanoparticle samples. Using this data along with the surface area approximated via TEM, the maximum surface

coverage of the control sample was calculated to be $3.1 \mu \mathrm{mol} / \mathrm{m}^{2}$, corresponding to a Zn sorption density of 1.9 sites $/ \mathrm{nm}^{2}$ and within the range of goethite site density values reported by Villalobos et al. (2003) and Hiemstra et al. (2010). The results also show that as aggregation state is increased, total uptake of $\mathrm{Zn}$ decreases while $\mathrm{Zn}$ retention increases, suggesting a shift in the predominant mode of $\mathrm{Zn}$ binding with increasing nanoparticle aggregation state.

The reduction in total $\mathrm{Zn}$ uptake with increased aggregation may be due to the decrease in readily-accessible external surface area associated with aggregation in suspension, while the greater extent of $\mathrm{Zn}$ retention with increased aggregation indicates that the metal ions associated with the aggregated particles are bound more irreversibly than to the dispersed control nanoparticles. This may be due to the creation of interparticle pore space between the nanoparticles during aggregation (GILBERT et al., 2007) which, while more difficult to initially access, may trap dissolved zinc ions more securely within the aggregates. A study of $\mathrm{PbS}$ nanoparticle aggregates also suggests ion transport inhibition in the highly confined spaces between densely packed, aggregated nanocrystals, where selfdiffusion coefficients of water and ions decrease dramatically (LIU et al., 2009). Another possibility is that the oriented aggregation of nanoparticles preferentially increases the proportion of more strongly- 
binding sorption sites (PENN et al., 2006; WEIDLER et al., 1998; YUWONO et al., 2010) while simultaneously blocking or decreasing the proportion of more weakly-binding sorption sites. The macroscopic data in Figure 3 does not provide sufficient information to precisely determine the modes of sorption that are occurring and additional explanations may exist. The data do suggest, however, that $\mathrm{Zn}$ is bound to the iron oxyhydroxide nanoparticles in at least two different modes and that the proportions of these binding environments is changing with aggregation condition such that stronger or more interior-located binding sites dominate with increasing aggregation state.

\subsubsection{Spectroscopic results}

The experimental and fitted Zn K-edge EXAFS spectra and corresponding Fourier transforms (FT) for both the adsorbed and desorbed versions of the control and the $\mathrm{pH}$ - and ionic strengthaggregated samples show subtle changes in the raw EXAFS data, specifically differences in the amplitude and shape of the third and fourth oscillations $\left(\mathrm{k}=7-11 \AA^{-1}\right)$, that distinguish spectra of the adsorbed and desorbed samples (Figures 4, 5). The spectral changes between samples are more visible in the Fourier transforms, with the second-neighbor features appearing to shift to slightly longer distances upon desorption.

Fitting of the Zn EXAFS (Table 3) further reveals that the coordination numbers $(\mathrm{CN})$ for all shells of the desorbed samples are larger than those of the corresponding adsorbed samples. The consistency of this trend among the samples leads to sufficient confidence in the trend of the fit values despite the associated errors inherent in such fits. Additionally, the interatomic Zn-Fe distances appear to increase from an average of $3.19 \pm 0.03 \AA$ and $3.42 \pm 0.03 \AA$ among the adsorbed samples to $3.27 \pm$ $0.03 \AA$ and $3.46 \pm 0.03 \AA$ among the desorbed samples. These reported coordination number and interatomic distance ranges generally correlate with findings from previous studies (JUILLOT et al., 2008; LEE and ANDERSON, 2005; NACHTEGAAL and SPARKS, 2004; TRIVEDI et al., 2001). 
The larger Zn-Fe coordination numbers associated with the desorbed samples indicate that following the desorption of the weakly-sorbed zinc species, the more strongly-retained zinc is in a sorption environment containing, on average, a greater number of iron neighbors than when under the adsorbed conditions. This suggests the preferential removal of zinc associated with fewer iron atoms, such as those found in outer-sphere $(\mathrm{CN}=0)$ and mononuclear monodentate inner-sphere $(\mathrm{CN}=1)$ binding environments, and the retention of zinc in mononuclear bidentate inner-sphere $(\mathrm{CN}=2)$ environments, which are commonly associated with stronger binding (STUMM and MORGAN, 1996). An increased nanoparticle aggregation state seems to further favor the formation of these strong binding sites as discussed earlier based on the macroscopic data.

The increase in the $\mathrm{Zn}-\mathrm{O} \mathrm{CN}$ of the desorbed samples compared to the adsorbed samples is associated with a shift from zinc bound to less ordered (tetrahedrally coordinated) sites towards zinc bound to more ordered (octahedrally coordinated) sites, e.g. on goethite (JUILLOT et al., 2008). The ZnFe interatomic distances in the desorbed samples of 3.27 and $3.46 \AA$ are supportive of binuclear bidentate corner-sharing arrangements, as reported by Juillot et al. (2008) and Kim et al. (2008). Juillot assigns the $3.26 \AA$ distance to $\mathrm{Zn}$ binding to the [110] face of goethite and the $3.47 \AA$ distance to $\mathrm{Zn}$ binding to the [110]-like face of 2-line ferrihydrite. Studies have shown that the structure of iron oxyhydroxide nanoparticles, particularly at surfaces and edges, is particularly strained and distorted from their microscale counterparts (GILBERT et al., 2004; RUSTAD and FELMY, 2005), justifying the possibility of both binding arrangements on these nanoparticles.

Due to the similarity in the binding environments of the two distances reported by Juillot et al. (2008) and the documented disorder of nanoparticle surfaces, a simplified structural model was developed by representing a zinc surface sorption configuration as a bidentate corner-sharing arrangement to two edge-sharing $\mathrm{FeO}_{6}$ iron octahedral units and, using $\mathrm{Zn}-\mathrm{O}$ and $\mathrm{Zn}-\mathrm{Fe}$ distances determined experimentally through EXAFS fitting, applying the law of cosines accordingly: 


\section{$c^{2}=a^{2}+b^{2}-2 a b \cos \gamma$}

\section{(Equation 1)}

where $a, b, c=$ lengths of sides of a triangle

$\gamma=$ angle of triangle opposite side c

This allowed the calculation of the angle between the equatorial planes of the iron octahedra, assuming that an undistorted surface would yield a $180^{\circ}$ angle. Based on the EXAFS fitting results (Table 3), the increases in the two Zn-Fe distances observed between the adsorbed and desorbed samples correspond to a change in the equatorial planar angle from $163.5^{\circ}$ to $166.4^{\circ}$ (Figure 6) and from $172.6^{\circ}$ to $174.4^{\circ}$ (model not shown). These shifts denote a change upon desorption towards $\mathrm{Zn}$ retention to sites with a lower degree of surface disorder (i.e. an equatorial planar angle closer to $180^{\circ}$ ), indicating that such sites are more stable than their relaxed surface site counterparts. Further, using the range of angles between the equatorial planes of Fe octahedra calculated from the $\mathrm{Zn}-\mathrm{Fe} 1$ and $\mathrm{Zn}-\mathrm{Fe} 2$ shells to represent surface curvature, idealized ellipsoidal diameters of individual particles were calculated to range from 1.78 to $4.33 \mathrm{~nm}$, consistent with TEM observations from both this work and past studies (GuYODO et al., 2003).

\subsection{Copper sorption}

\subsubsection{Macroscopic results}

The macroscopic copper sorption data are presented in Figure 7, which shows the percentage of copper adsorbed following the uptake step and retained following the desorption step onto the various nanoparticle samples. Maximum surface coverage of the control sample was calculated to be 0.5 $\mu \mathrm{mol} / \mathrm{m}^{2}$, again indicating that surface precipitation is unlikely under these conditions. Whether 
aggregation is induced by $\mathrm{pH}$ or ionic strength, both copper adsorption and copper retention decrease with increased aggregation, although the differences between control and aggregated samples as well as across various aggregation states are relatively modest.

The results of the copper macroscopic uptake/desorption experiments suggest that a single consistent sorption mode dominates the uptake behavior of copper due to the relative lack of difference in $\mathrm{Cu}(\mathrm{II})$ retention between the adsorbed and desorbed states of the experiment. This consistently small change along with copper sorption behavior being generally independent of aggregation state indicates that the binding mode for copper is consistently relatively strong (e.g. inner-sphere). The lack of significant desorption may also be attributed to the $\mathrm{pH}$ change of only one unit (as opposed to two units for the zinc samples), from 6.0 to 5.0; further decreases in $\mathrm{pH}$ run the risk of nanoparticle dissolution which would complicate the sorption studies, and were therefore not conducted.

\subsubsection{Spectroscopic results}

The experimental and fitted $\mathrm{Cu}$ K-edge EXAFS spectra and corresponding Fourier transforms for both the adsorbed and desorbed versions of the control sample and the samples aggregated at $1.0 \mathrm{M}$ ionic strength and pH 10 are shown in Figure 8. Due primarily to the lower degree of variability in the macroscopic data as a function of $\mathrm{pH}$ and ionic strength, only the most strongly aggregated samples were analyzed by EXAFS spectroscopy. Qualitative differences between the adsorbed and desorbed samples include the shoulder features visible in the EXAFS at $\mathrm{k}=7.75$ and $10.75 \AA^{-1}$ which become less prominent among the desorbed sample spectra. This change is also evident in the FT as reduced magnitudes (peak heights) of the second-neighbor features. No significant differences can be observed in the first-neighbor feature between the adsorbed and desorbed samples.

The EXAFS fit results (Table 4) confirm the qualitative observations of the raw EXAFS spectra and Fourier transforms. The interatomic distances for all neighbors remain essentially constant within 
the associated errors. This suggests that no significant change in the sorption mode exists between $\mathrm{Cu}$ in the adsorbed and desorbed environments, which is consistent with the relatively small amount of $\mathrm{Cu}$ desorption that occurred across all samples (Figure 7). In all cases the first shell is fit as a $\mathrm{Cu}-\mathrm{O}$ scattering interaction with a coordination number between $3.1-3.3 \pm 0.3$ at an average distance of 1.95 $\pm 0.01 \AA$ A Although copper is typically hexa-aquo coordinated in solution, Jahn-Teller distortion of the $\mathrm{Cu}\left(\mathrm{H}_{2} \mathrm{O}\right)_{6}$ octahedron results in the first shell being dominated by scattering from the four equatorial oxygens (COTTON and WiLKInSON, 1988). These fitting results are in agreement with prior room temperature studies of $\mathrm{Cu}$ (II) sorption to mineral surfaces (ALCACIO et al., 2001; GILBERT et al., 2009; LIN et al., 2004b).

Slight decreases in $\mathrm{CN}$ of the $\mathrm{Cu}-\mathrm{Fe}$ neighbors after the desorption step occur in all samples and agree with the visual reduction in magnitude of those features in the Fourier transforms. This decrease may be attributable to contributions from the fraction of $\mathrm{Cu}(\mathrm{II})_{(\mathrm{aq})}$ that either returns to solution and/or persists as outer-sphere sorption complexes as copper is removed from the surfaces of the iron oxyhydroxides; importantly, such species would still retain a similar first-shell $\mathrm{Cu}-\mathrm{O}$ configuration as described above, limiting $\mathrm{CN}$ changes to only the second-shell features. This trend also supports the existence of a single local binding environment that dominates in both adsorption and desorption samples, in agreement with the macroscopic data. The fitting results identified two second-neighbor $\mathrm{Cu} / \mathrm{Fe}$ shells at 3.03 and $3.28 \AA$, consistent with those reported by other investigators (LIN et al., 2004a; MoOn and PeAcock, 2012; PEACOCK and Sherman, 2004; SCHEINOST et al., 2001) and associated with copper sorption in inner-sphere mononuclear bidentate edge-sharing and corner-sharing arrangements. The $\mathrm{Cu}-\mathrm{Fe}$ coordination numbers are statistically equal at these two distances, suggesting roughly equal proportions of edge- and corner-sharing sorption complexes.

Elemental identification of the second neighbors has proven difficult, as many investigators have reported that attempts to identify them as either $\mathrm{Cu}$ or Fe have been inconclusive (ALCACIO et al., 
2001; PARKMAN et al., 1999). Activity diagrams generated using Geochemists’ Workbench® (not shown) indicated that the formation of $\mathrm{Cu}$-hydroxy clusters, $\mathrm{Cu}$ dimers, or $\mathrm{Cu}$ precipitates is unlikely given the $\mathrm{pH}$ range of these experiments, pointing to bidentate sorption complexes as the most likely mode of $\mathrm{Cu}(\mathrm{II})$ uptake onto the particles.

\section{CONCLUSIONS}

The combination of macroscopic sorption/desorption experiments with detailed spectroscopic analysis provides useful insights into the modes of metal ion uptake and retention to iron oxyhydroxide nanoparticles and the effects of aggregation on sorption behavior. From these studies, zinc appears to be sorbed in at least two different configurations on the nanoparticles and/or their associated aggregates, as demonstrated by the macroscopic and spectroscopic differences observed between the adsorbed and desorbed samples. Specifically, the weaker of the sorption modes (e.g. monodentate, outer-sphere complexes) is preferentially removed during desorption, leaving behind the more strongly bound species (e.g. bidentate, structurally-substituted complexes).

The EXAFS data documents shifts of the Zn-Fe distance towards Fe-Fe distances found in goethite of $3.28 \AA$ and $3.46 \AA$ after desorption, suggesting that the retained zinc is found at more ordered and strongly-binding surface sites of the iron oxyhydroxide aggregates and even introducing the possibility of some degree of structural incorporation within the nanoparticle near-surface regime. Overall, greater extents of aggregation, whether by $\mathrm{pH}$ or ionic strength, appear to have improved the effectiveness of the aggregates to retain zinc by increasing the proportion of strong sorption sites, favoring the formation of bidentate corner-sharing inner-sphere complexes, and/or limiting zinc desorption through the retention of zinc ions in interparticle pore spaces.

In contrast, copper sorption appears to be occurring in a consistent sorption configuration within the experimental parameters. This is supported by the relatively small degree of desorption and the 
similarity in uptake/retention amongst the control and the aggregated samples. Decreases in $\mathrm{Cu}-\mathrm{Fe}$ coordination values following desorption and the constant $\mathrm{Cu}-\mathrm{O}$ and $\mathrm{Cu}-\mathrm{Fe}$ interatomic distances in the spectroscopic data also indicate that copper sorption is dominated by a constant binding configuration. The $\mathrm{Cu}$ - Fe distances were determined to be approximately $3.03 \AA$ and $3.28 \AA$ from the EXAFS data, which is best explained by inner-sphere mononuclear bidentate edge- and corner-sharing surface complexes. Generally, the extent of copper binding appears to be largely unaffected by aggregation and is bound equally well under all solution conditions, also suggesting comparable sorption strengths between the two sorption complexes within the parameters examined.

In conclusion, zinc and copper exhibit fundamentally different sorption behaviors to iron oxyhydroxide nanoparticles under the experimental conditions examined, with nanoparticle aggregation causing varying effects on the sorption and retention behavior of each metal. This is likely due to a greater diversity of potential $\mathrm{Zn}$ sorption sites (and corresponding sorption strengths) compared to $\mathrm{Cu}$ sorption sites, resulting in more complex $\mathrm{Zn}$ sorption behavior and more uniform $\mathrm{Cu}$ sorption behavior as a function of aggregation state. The data also indicate that two distinct methods of aggregation, $\mathrm{pH}$ and ionic strength, appear to induce similar changes on the macroscopic metal uptake/retention behavior of our nanoparticle aggregates. This can be noted by the parallel effects of $\mathrm{pH}$ and ionic strength on an individual metal ion's sorption, as documented in the macroscopic data, and the similarity in the extent of aggregation as shown in the TEM images. This is likely because the method of aggregation is essentially identical, resulting in rapidly-formed aggregates with open structures and containing significant amounts of interstitial water, compared with drying or freezing in which interstitial water is largely removed, creating tightly packed aggregates and strong orientation. Studying such effects is important to understanding their implications on both the natural attenuation processes of metal-contaminated environments as well as the development of engineered strategies for the removal of potentially toxic dissolved metals from aqueous systems. 


\section{ACKNOWLEDGMENTS}

This work was funded by the National Science Foundation, Division of Earth Sciences, Grant \#061821711, Cottrell College Science Award \#6940/6912 from the Research Corporation, and a Henry Dreyfus Teacher-Scholar Award. Portions of this research were carried out at the Stanford Synchrotron Radiation Lightsource, a national user facility operated by Stanford University on behalf of the U.S. Department of Energy, Office of Basic Energy Sciences. The authors thank Dr. Jian-Guo Zheng (UC Irvine) for lending his expertise in collecting TEM data, Dr. Fettah Kosar (Harvard University) and Dr. Jeffrey Cohlberg (CSU-Long Beach) for assistance with DLS, Suzie Shdo for assisting with DLS data collection, and Rebecca Chesne for collecting and processing the XRD data and additional DLS data. Thanks also to Lauryn DeGreeff, Chris Lentini, Brian Reinsch and other members of the Chapman University Environmental Geochemistry Lab for their earlier studies on metal uptake and iron oxyhydroxide nanoparticle aggregation. 


\section{References}

Alcacio, T. E., Hesterberg, D., Chou, J. W., Martin, J. D., Beauchemin, S., and Sayers, D. E., 2001. Molecular scale characteristics of $\mathrm{Cu}$ (II) bonding in goethite-humate complexes. Geochim. Cosmochim. Acta 65, 1355-1366.

Banfield, J. F., Welch, S. A., Zhang, H. Z., Ebert, T. T., and Penn, R. L., 2000. Aggregation-based crystal growth and microstructure development in natural iron oxyhydroxide biomineralization products. Science 289, 751-754.

Benjamin, M. M. and Leckie, J. O., 1981. Multiple-site adsorption of Cd, Cu, Zn, and $\mathrm{Pb}$ on amorphous iron oxyhydroxide. Journal of Colloid \& Interface Science 79, 209-221.

Bethke, C. M., 1996. Geochemical Reaction Modelling: Concepts and Applications. Oxford University Press, New York, NY.

Bethke, C. M., 2002. The Geochemists Workbench Version 4.0: A User's Guide. University of Illinois, Urbana, IL.

Blowes, D. W., Ptacek, C. J., Jambor, J. L., Weisener, C. G., Heinrich, D. H., and Karl, K. T., 2003. The Geochemistry of Acid Mine Drainage, Treatise on Geochemistry. Pergamon, Oxford.

Burleson, D. J. and Penn, R. L., 2006. Two-step growth of goethite from ferrihydrite. Langmuir 22, 402-409.

Cotton, F. A. and Wilkinson, G., 1988. Advanced Inorganic Chemistry. John Wiley and Sons, New York, NY.

Cwiertny, D. M., Handler, R. M., Schaefer, M. V., Grassian, V. H., and Scherer, M. M., 2008. Interpreting nanoscale size-effects in aggregated Fe-oxide suspensions: reaction of Fe(II) with goethite. Geochim. Cosmochim. Acta 72, 1365-1380.

Druschel, G. K., Baker, B. J., Gihring, T. M., and Banfield, J. F., 2004. Acid mine drainage biogeochemistry at Iron Mountain, California. Geochemical Transactions 5, 13-32.

Dyer, J. A., Trivedi, P., Scrivner, N. C., and Sparks, D. L., 2004. Surface complexation modeling of zinc sorption onto ferrihydrite. Journal of Colloid and Interface Science 270, 56-65.

Dzombak, D. A. and Morel, F. M. M., 1990. Surface complexation modeling: hydrous ferric oxide. Wiley-Interscience, New York.

Ford, R. G., Scheinost, a. C., and Sparks, D. L., 2001. Frontiers in metal sorption/precipitation mechanisms on soil mineral surfaces, Advances in Agronomy. Academic Press.

Gilbert, B., Huang, F., Zhang, H. Z., Waychunas, G. A., and Banfield, J. F., 2004. Nanoparticles: Strained and stiff. Science 305, 651-654.

Gilbert, B., Lu, G. P., and Kim, C. S., 2007. Stable cluster formation in aqueous suspensions of iron oxyhydroxide nanoparticles. Journal of Colloid and Interface Science 313, 152-159.

Gilbert, B., Ono, R. K., Ching, K. A., and Kim, C. S., 2009. The effects of nanoparticle aggregation processes on aggregate structure and metal uptake. Journal of Colloid and Interface Science 339, 285-295.

Grafe, M., Nachtegaal, M., and Sparks, D. L., 2004. Formation of metal-arsenate precipitates at the goethite-water interface. Environmental Science \& Technology 38, 6561-6570.

Guyodo, Y., Mostrom, A., Penn, R. L., and Banerjee, S. K., 2003. From Nanodots to Nanorods: Oriented aggregation and magnetic evolution of nanocrystalline goethite. Geophys. Res. Lett $30,1512$.

Herbert Jr, R. B., 1996. Metal retention by iron oxide precipitation from acidic ground water in Dalarna, Sweden. Applied Geochemistry 11, 229-235. 
Hiemstra, T., Antelo, J., Rahnemaie, R., and van Riemsdijk, W. H., 2010. Nanoparticles in natural systems I: The effective reactive surface area of the natural oxide fraction in field samples. Geochim. Cosmochim. Acta 74, 41-58.

Hiemstra, T. and van Riemsdijk, W. H., 1996. A surface structural approach to ion adsorption: the charge distribution (CD) model. Journal of Colloid and Interface Science 179, 488-508.

Hou, T., Xu, R. K., Tiwari, D., and Zhao, A. Z., 2007. Interaction between electrical double layers of soil colloids and Fe/Al oxides in suspensions. Journal of Colloid and Interface Science 310, 670-674.

Jamieson, H. E., Robinson, C., Alpers, C. N., McCleskey, R. B., Nordstrom, D. K., and Peterson, R. C., 2005. Major and trace element composition of copiapite-group minerals and coexisting water from the Richmond mine, Iron Mountain, California. Chemical Geology 215, 387-405.

Jansen, E., Kyek, A., Schafer, W., and Schwertmann, U., 2002. The structure of six-line ferrihydrite. Appl Phys a-Mater 74, S1004-S1006.

Juillot, F., Maréchal, C., Ponthieu, M., Cacaly, S., Morin, G., Benedetti, M., Hazemann, J. L., Proux, O., and Guyot, F., 2008. Zn isotopic fractionation caused by sorption on goethite and 2-Lines ferrihydrite. Geochim. Cosmochim. Acta 72, 4886-4900.

Kim, C. S., Lentini, C. J., and Waychunas, G. A., 2008. Synchrotron-based studies of metal adsorption and structural incorporation with iron oxyhydroxide nanoparticles. In: Barnett, M. O. (Ed.), Adsorption of Metals By Geomedia II: Variables, Mechanisms, and Model Applications. Elsevier Academic Press.

Lee, S. and Anderson, P. R., 2005. EXAFS study of Zn sorption mechanisms on hydrous ferric oxide over extended reaction time. Journal of Colloid and Interface Science 286, 82-89.

Li, S. Z. and Xu, R. K., 2008. Electrical double layers' interaction between oppositely charged particles as related to surface charge density and ionic strength. Colloid Surf. A-Physicochem. Eng. Asp. 326, 157-161.

Lin, S. H., Kao, H. C., Cheng, C. H., and Juang, R. S., 2004a. An EXAFS study of the structures of copper and phosphate sorbed onto goethite. Colloid Surf. A-Physicochem. Eng. Asp. 234, 7175.

Lin, S. H., Kao, H. C., Cheng, C. H., and Juang, R. S., 2004b. An EXFAS study of the structures of copper and phosphate sorbed onto goethite. Colloids and Surfaces a-Physicochemical and Engineering Aspects 234, 71-75.

Liu, J., Aruguete, D. M., Murayama, M., and Hochella, M. F., Jr., 2009. Influence of Size and Aggregation on the Reactivity of an Environmentally and Industrially Relevant Nanomaterial (PbS). Environmental Science \& Technology 43, 8178-8183.

Lowry, G. V., Shaw, S., Kim, C. S., Rytuba, J. J., and Brown, G. E. J., 2004. Macroscopic and microscopic observations of particle-facilitated mercury transport from New Idria and Sulphur Bank mercury mine tailings. Environmental Science \& Technology 38, 5101-5111.

Madden, A. S. and Hochella, M. F., Jr., 2005. A test of geochemical reactivity as a function of mineral size: Manganese oxidation promoted by hematite nanoparticles. Geochim. Cosmochim. Acta 69, 389-398.

Moon, E. M. and Peacock, C. L., 2012. Adsorption of Cu(II) to ferrihydrite and ferrihydrite-bacteria composites: Importance of the carboxyl group for $\mathrm{Cu}$ mobility in natural environments.

Geochim. Cosmochim. Acta 92, 203-219.

Nachtegaal, M. and Sparks, D. L., 2004. Effect of iron oxide coatings on zinc sorption mechanisms at the clay-mineral/water interface. Journal of Colloid and Interface Science 276, 13-23. 
Nordstrom, D. K. and Alpers, C. N., 1999. Negative pH, efflorescent mineralogy, and consequences for environmental restoration at the Iron Mountain Superfund site, California. Proceedings of the National Academy of Science 96, 7.

Nordstrom, D. K., Alpers, C. N., Ptacek, C. J., and Blowes, D. W., 2000. Negative pH and extremely acidic mine waters from Iron Mountain, California. Environmental Science \& Technology 34, 254-258.

Parkman, R. H., Charnock, J. M., Bryan, N. D., Livens, F. R., and Vaughan, D. J., 1999. Reactions of copper and cadmium ions in aqueous solution with goethite, lepidocrocite, mackinawite, and pyrite. American Mineralogist 84, 407-419.

Peacock, C. L. and Sherman, D. M., 2004. Copper(II) sorption onto goethite, hematite and lepidocrocite: A surface complexation model based on ab initio molecular geometries and EXAFS spectroscopy. Geochim. Cosmochim. Acta 68, 2623-2637.

Penn, R. L., 2004. Kinetics of oriented aggregation. Journal of Physical Chemistry B 108, 1270712712.

Penn, R. L., Erbs, J. J., and Gulliver, D. M., 2006. Controlled growth of alpha-FeOOH nanorods by exploiting oriented aggregation. Journal of Crystal Growth 293, 1-4.

Penn, R. L., Zhu, C., Xu, H., and Veblen, D. R., 2001. Iron oxide coatings on sand grains from the Atlantic coastal plain: High-resolution transmission electron microscopy characterization. Geology 29, 843-846.

Rehr, J. J., Leon, J. M. d., Zabinsky, S. I., and Albers, R. C., 1991. Theoretical X-ray absorption finestructure standards. Journal of the American Chemical Society 113, 5135-5140.

Rustad, J. R. and Felmy, A. R., 2005. The influence of edge sites on the development of surface charge on goethite nanoparticles: A molecular dynamics investigation. Geochim. Cosmochim. Acta 69, 1405-1411.

Scheinost, A. C., Abend, S., Pandya, K. I., and Sparks, D. L., 2001. Kinetic controls on Cu and Pb sorption by ferrihydrite. Environmental Science \& Technology 35, 1090-1096.

Schwertmann, U. and Cornell, R. M., 2000. Iron Oxides in the Laboratory: Preparation and Characterization. Wiley-VCH, Toronto.

Stern, E. A. and Heald, S. M., 1979. X-ray filter assembly for fluorescence measurements of x-ray absorption fine structure. Review of Scientific Instruments 50, 1579-1582.

Stumm, W., 1997. Reactivity at the mineral-water interface: Dissolution and inhibition. Colloid Surf. A-Physicochem. Eng. Asp. 120, 143-166.

Stumm, W. and Morgan, J. J., 1996. Aquatic Chemistry. John Wiley \& Sons, New York, NY.

Trivedi, P., Axe, L., and Tyson, T. A., 2001. An analysis of zinc sorption to amorphous versus crystalline iron oxides using XAS. Journal of Colloid and Interface Science 244, 230-238.

van der Zee, C. and Roberts, D. R., 2003. Nanogoethite is the dominant reactive oxyhydroxide phase in lake and marine sediments. Geology 31, 993-996.

Villalobos, M., Cheney, M. A., and Alcaraz-Cienfuegos, J., 2009. Goethite surface reactivity: II. A microscopic site-density model that describes its surface area-normalized variability. Journal of Colloid and Interface Science 336, 412-422.

Villalobos, M., Trotz, M. A., and Leckie, J. O., 2003. Variability in goethite surface site density: evidence from proton and carbonate sorption. Journal of Colloid \& Interface Science 268, 273287.

Waychunas, G. A. and Brown, G. E., Jr., 1994. Fluorescence yield XANES and EXAFS experiments: application to highly dilute and surface samples. Advances in X-Ray Analysis 37, 607-617.

Webb, S. M., 2005. SIXpack: a graphical user interface for XAS analysis using IFEFFIT. Phys. Scr. T115, 1011-1014. 
Weidler, P. G., Hug, S. J., Wetche, T. P., and Hiemstra, T., 1998. Determination of growth rates of (100) and (110) faces of synthetic goethite by scanning force microscopy. Geochim. Cosmochim. Acta 62, 3407-3412.

Xu, Y., Axe, L., Yee, N., and Dyer, J. A., 2006. Bidentate complexation modeling of heavy metal adsorption and competition on goethite. Environmental Science \& Technology 40, 2213-2218.

Yuwono, V. M., Burrows, N. D., Soltis, J. A., and Penn, R. L., 2010. Oriented aggregation: formation and transformation of mesocrystal intermediates revealed. Journal of the American Chemical Society 132, 2163-2165.

Yuwono, V. M., Burrows, N. D., Soltis, J. A., Tram Anh, D., and Penn, R. L., 2012. Aggregation of ferrihydrite nanoparticles in aqueous systems. Faraday Discussions 159, 235-245. 


\section{TABLE CAPTIONS}

Table 1. Aggregation conditions and key parameters of control and nanoparticle aggregates.

Table 2. Average particle/aggregate hydrodynamic diameter $\left(\mathrm{d}_{\mathrm{p}}\right)$ and polydispersity index (PDI) for all samples at various stages during the adsorption/desorption process. Standard deviations are shown below averages.

Table 3. Zn K-edge EXAFS fitting results (see Figs. 4-5 for corresponding EXAFS spectra and Fourier transforms), including coordination numbers $(\mathrm{CN})$, interatomic distances $(\mathrm{R})$, Debye-Waller factors $\left({ }^{2}\right)$, energy shift $\left(\mathrm{E}_{0}\right)$, and goodness of fit ( $\mathrm{R}$ factor). All $\mathrm{S}_{0}$ values were held constant at 0.9 . ${ }^{*}$ Debye-Waller values held constant.

Table 4. Cu K-edge EXAFS fitting results (see Fig. 8 for corresponding EXAFS spectra and Fourier transforms), including coordination numbers $(\mathrm{CN})$, interatomic distances $(\mathrm{R})$, Debye-Waller factors $\left({ }^{2}\right)$, energy shift $\left(\mathrm{E}_{0}\right)$, and goodness of fit ( $\mathrm{R}$ factor). All $\mathrm{S}_{0}$ values were held constant at 0.9 . ${ }^{*}$ Debye-Waller values held constant. 


\section{Table 1.}

$\begin{array}{cccc}\text { Sample ID } & \text { pH } & \begin{array}{c}\text { Ionic } \\ \text { Strength }\end{array} & \begin{array}{c}\text { BET Surface } \\ \text { Area }\left(\mathrm{m}^{2} / \mathrm{g}\right)\end{array} \\ \text { pH 10 } & 10.0 & 0.001 \mathrm{M} & 391 \pm 32 \\ \text { pH 8 } & 8.0 & 0.001 \mathrm{M} & 349 \pm 3 \\ \text { Control } & 5.0 & 0.001 \mathrm{M} & 306 \pm 13 \\ \text { IS 0.1 M } & 5.0 & 0.100 \mathrm{M} & 318 \pm 10 \\ \text { IS 1.0 M } & 5.0 & 1.000 \mathrm{M} & 299 \pm 7\end{array}$


Table 2.

\begin{tabular}{|c|c|c|c|c|c|c|}
\hline \multirow[t]{2}{*}{ Sample } & \multicolumn{2}{|c|}{ Post re-equilibration } & \multicolumn{2}{|c|}{ Adsorption } & \multicolumn{2}{|c|}{ Desorption } \\
\hline & $d_{p}(n m)$ & PDI & $d_{p}(n m)$ & PDI & $d_{p}(n m)$ & PDI \\
\hline pH 10 & 5500 & 0.76 & 5100 & 0.50 & 1700 & 0.60 \\
\hline Std. dev. & 1013 & 0.12 & 508 & 0.11 & 133 & 0.02 \\
\hline pH 8 & 1000 & 0.45 & 4000 & 1.15 & 1000 & 0.48 \\
\hline Std. dev. & 114 & 0.04 & 363 & 0.03 & 117 & 0.04 \\
\hline Control & 600 & 0.31 & 1200 & 0.46 & 800 & 0.38 \\
\hline Std. dev. & 8 & 0.01 & 50 & 0.02 & 33 & 0.01 \\
\hline IS $0.1 \mathrm{M}$ & 5900 & 1.18 & 4400 & 0.75 & 1500 & 0.57 \\
\hline Std. dev. & 335 & 0.32 & 172 & 0.04 & 183 & 0.02 \\
\hline IS $1.0 \mathrm{M}$ & 6400 & 0.71 & 6400 & 1.19 & 6300 & 0.86 \\
\hline Std. dev. & N/A & N/A & 367 & 0.17 & 97 & 0.35 \\
\hline
\end{tabular}


Table 3.

\begin{tabular}{|c|c|c|c|c|c|c|c|c|c|c|c|c|}
\hline $\begin{array}{l}\text { Aggregation } \\
\text { Condition }\end{array}$ & Sorption Step & \multicolumn{3}{|c|}{$\mathrm{Zn}-\mathrm{O}$} & \multicolumn{3}{|c|}{$\mathrm{Zn}-\mathrm{Fe} 1$} & \multicolumn{3}{|c|}{$\mathrm{Zn}-\mathrm{Fe} 2$} & $\begin{array}{c}\text { E0 } \\
\text { shift }\end{array}$ & $\begin{array}{c}\mathrm{R} \\
\text { factor }\end{array}$ \\
\hline IS $1.0 \mathrm{M}$ & Adsorption & $3.8(2)$ & $1.98(1)$ & $0.008(1)$ & $0.8(2)$ & $3.18(3)$ & 0.01 & $0.9(3)$ & $3.41(3)$ & 0.01 & $3.7(7)$ & 0.008 \\
\hline IS $1.0 \mathrm{M}$ & Desorption & $4.4(3)$ & $1.99(1)$ & $0.010(1)$ & $1.2(3)$ & $3.27(3)$ & 0.01 & $1.8(4)$ & $3.46(3)$ & 0.01 & $3.4(8)$ & 0.0112 \\
\hline IS $0.1 \mathrm{M}$ & Adsorption & $3.8(2)$ & $1.98(1)$ & $0.008(1)$ & $0.9(2)$ & $3.18(2)$ & 0.01 & $1.0(3)$ & $3.41(2)$ & 0.01 & $3.8(7)$ & 0.0072 \\
\hline Control & Adsorption & $3.8(2)$ & $1.98(1)$ & $0.008(1)$ & $0.8(2)$ & $3.18(3)$ & 0.01 & $0.9(3)$ & $3.41(3)$ & 0.01 & $3.7(7)$ & 0.008 \\
\hline Control & Desorption & $4.5(2)$ & 1.99(1) & $0.010(1)$ & $1.1(3)$ & $3.26(3)$ & 0.01 & $1.3(3)$ & $3.45(3)$ & 0.01 & $4.3(7)$ & 0.0085 \\
\hline pH 8 & Adsorption & $4.0(2)$ & $1.98(1)$ & $0.009(1)$ & $1.0(2)$ & $3.21(2)$ & 0.01 & $1.0(3)$ & $3.45(3)$ & 0.01 & $3.8(7)$ & 0.0092 \\
\hline pH 8 & Desorption & $4.6(3)$ & 2.01(1) & $0.012(1)$ & $1.1(2)$ & $3.29(3)$ & 0.01 & $1.2(3)$ & $3.49(3)$ & 0.01 & $3.9(7)$ & 0.009 \\
\hline
\end{tabular}


Table 4.

\begin{tabular}{|c|c|c|c|c|c|c|c|c|c|c|c|c|}
\hline \multirow{2}{*}{$\begin{array}{l}\text { Aggregation } \\
\text { Condition }\end{array}$} & \multirow[b]{2}{*}{ Sorption Step } & \multicolumn{3}{|c|}{$\mathrm{Cu}-\mathrm{O}$} & \multicolumn{3}{|c|}{$\mathrm{Cu}-\mathrm{Fe}$} & \multicolumn{3}{|c|}{$\mathrm{Cu}-\mathrm{Fe}$} & \multirow[b]{2}{*}{$\mathrm{E}_{0}$ shift } & \multirow{2}{*}{$\begin{array}{c}\mathrm{R} \\
\text { factor }\end{array}$} \\
\hline & & $\mathrm{CN}$ & $\mathrm{R}(\AA)$ & ${ }^{2}\left(\AA^{2}\right)$ & $\mathrm{CN}$ & $\mathrm{R}(\AA)$ & ${ }^{2}\left(\AA^{2}\right)^{*}$ & $\mathrm{CN}$ & $\mathrm{R}(\AA)$ & $\begin{array}{c}\sigma^{2} \\
\left(\AA^{2}\right)^{*}\end{array}$ & & \\
\hline IS $1.0 \mathrm{M}$ & Adsorption & $3.2(3)$ & $1.95(1)$ & $0.004(1)$ & $2.2(5)$ & $3.02(2)$ & 0.01 & $2.2(6)$ & $3.29(2)$ & 0.01 & $1.0(1.3)$ & 0.0344 \\
\hline IS $1.0 \mathrm{M}$ & Desorption & $3.2(2)$ & $1.96(1)$ & $0.004(1)$ & $1.6(4)$ & $3.04(2)$ & 0.01 & $1.9(5)$ & $3.27(2)$ & 0.01 & $0.5(1.0)$ & 0.0226 \\
\hline Control & Adsorption & $3.3(3)$ & $1.95(1)$ & $0.003(1)$ & $2.0(4)$ & $3.03(2)$ & 0.01 & $2.3(6)$ & $3.30(2)$ & 0.01 & $0.6(1.4)$ & 0.033 \\
\hline Control & Desorption & $3.2(2)$ & $1.96(1)$ & $0.004(1)$ & $1.9(4)$ & $3.04(2)$ & 0.01 & $2.2(5)$ & $3.28(2)$ & 0.01 & $0.9(1.0)$ & 0.0222 \\
\hline pH 10 & Adsorption & $3.1(2)$ & $1.95(1)$ & $0.003(1)$ & $2.2(4)$ & $3.03(2)$ & 0.01 & $2.4(6)$ & $3.30(2)$ & 0.01 & $0.6(1.2)$ & 0.0261 \\
\hline $\mathrm{pH} 10$ & Desorption & $3.2(3)$ & $1.96(1)$ & $0.004(1)$ & $1.9(4)$ & $3.03(2)$ & 0.01 & $2.0(6)$ & $3.28(2)$ & 0.01 & $0.8(1.2)$ & 0.0292 \\
\hline
\end{tabular}




\section{FIGURE CAPTIONS}

Figure 1. Powder X-ray diffraction patterns of the control (unaggregated) iron oxyhydroxide nanoparticles and nanoparticles that have been aggregated at increasing ionic strength and $\mathrm{pH}$. Ferrihydrite (solid gray lines (JANSEN et al., 2002)) and goethite (dashed lines, No. 29-0713) PDFs are included for comparison.

Figure 2. Representative TEM images of iron oxyhydroxide nanoparticles a) following aggregation at $\mathrm{pH} 10$, with selected gaps in the aggregate outlined; b) in the control (non-aggregated) state, with arrows or circles indicating individual particles; and c) following aggregation at $1.0 \mathrm{M}$ ionic strength, where the lines highlight the lattice fringes indicating crystallinity. Higher magnification images of each sample are shown directly below each of the three images.

Figure 3. Macroscopic uptake/retention results indicating the percentage of introduced zinc initially adsorbed to the various nanoparticle samples and the percentage of introduced zinc retained to the same samples following desorption. Standard deviations based on triplicates of the control uptake/retention experiments were $1.1 \%$ and $1.4 \%$, respectively.

Figure 4. Zinc K-edge EXAFS spectra and Fourier transforms (black) with overlain fits (gray) for both the adsorbed and desorbed versions of the control and $\mathrm{pH}$-aggregated samples.

Figure 5. Zinc K-edge EXAFS spectra and Fourier transforms (black) with overlain fits (gray) for both the adsorbed and desorbed versions of the control and ionic strength-aggregated samples.

Figure 6. Simplified geometrical interpretation of the Zn EXAFS fitting results, with sorbed $\mathrm{Zn}$ forming a bidentate corner-sharing inner-sphere sorption complex to two edge-sharing iron octahedra. The observed increase in Zn-Fe interatomic distance can be correlated to a lower degree of distortion between the two iron octahedra's equatorial planes, as indicated by the closer resemblance to the $180^{\circ}$ idealized model found in goethite and consistent with a greater degree of bond stability.

Figure 7. Macroscopic uptake/retention results indicating the percentage of introduced copper initially adsorbed to the various nanoparticle samples and the percentage of copper retained to the same samples following desorption. Standard deviations based on triplicates of the control uptake/retention experiments were $0.8 \%$ and $2.2 \%$, respectively.

Figure 8. Copper K-edge EXAFS spectra and Fourier transforms (black) with overlain fits (gray) for both the adsorbed and desorbed versions of the control and samples aggregated at $\mathrm{pH} 10$ and $1.0 \mathrm{M}$ ionic strength. 


\section{FIGURES}

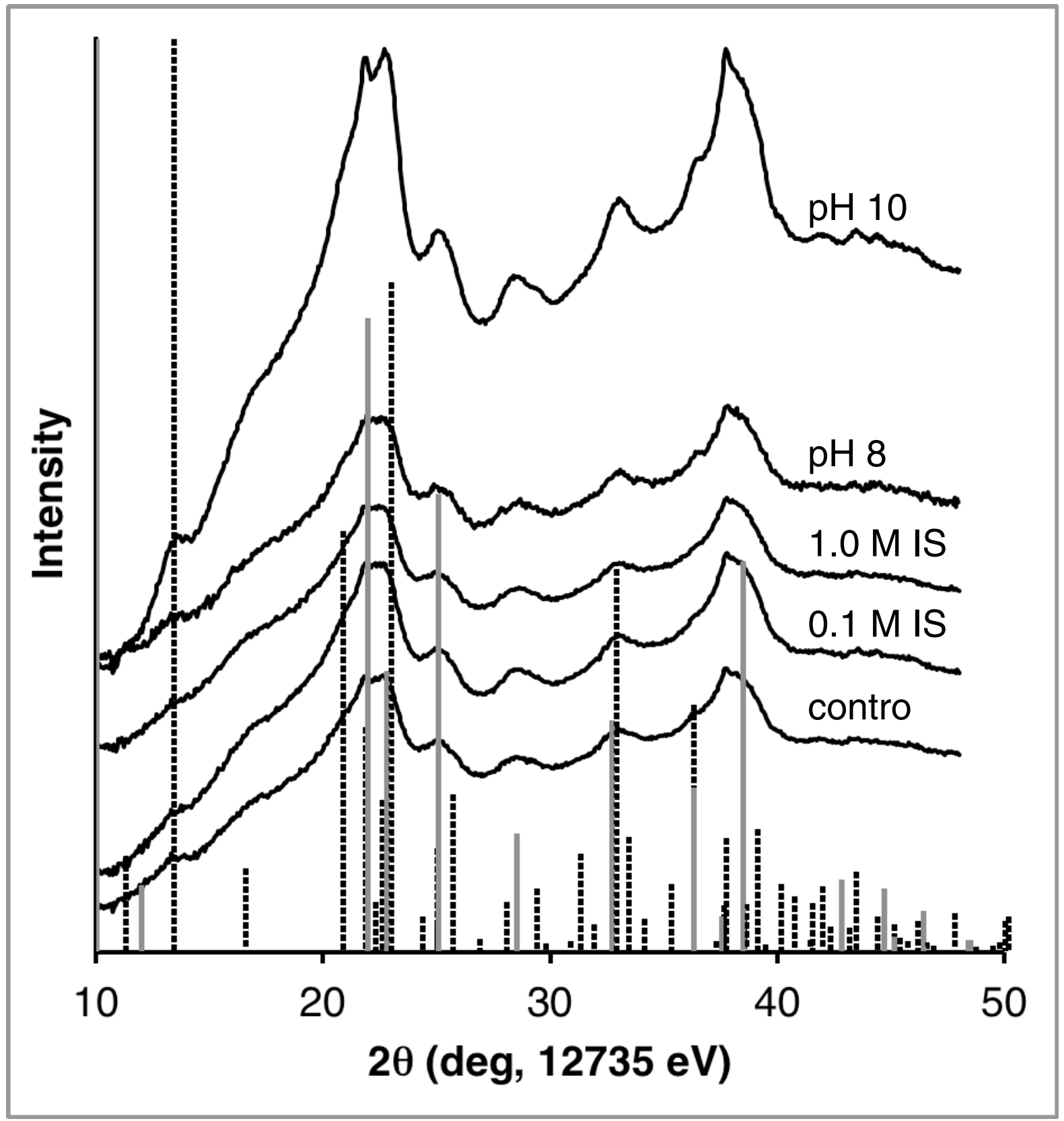

Figure 1. 


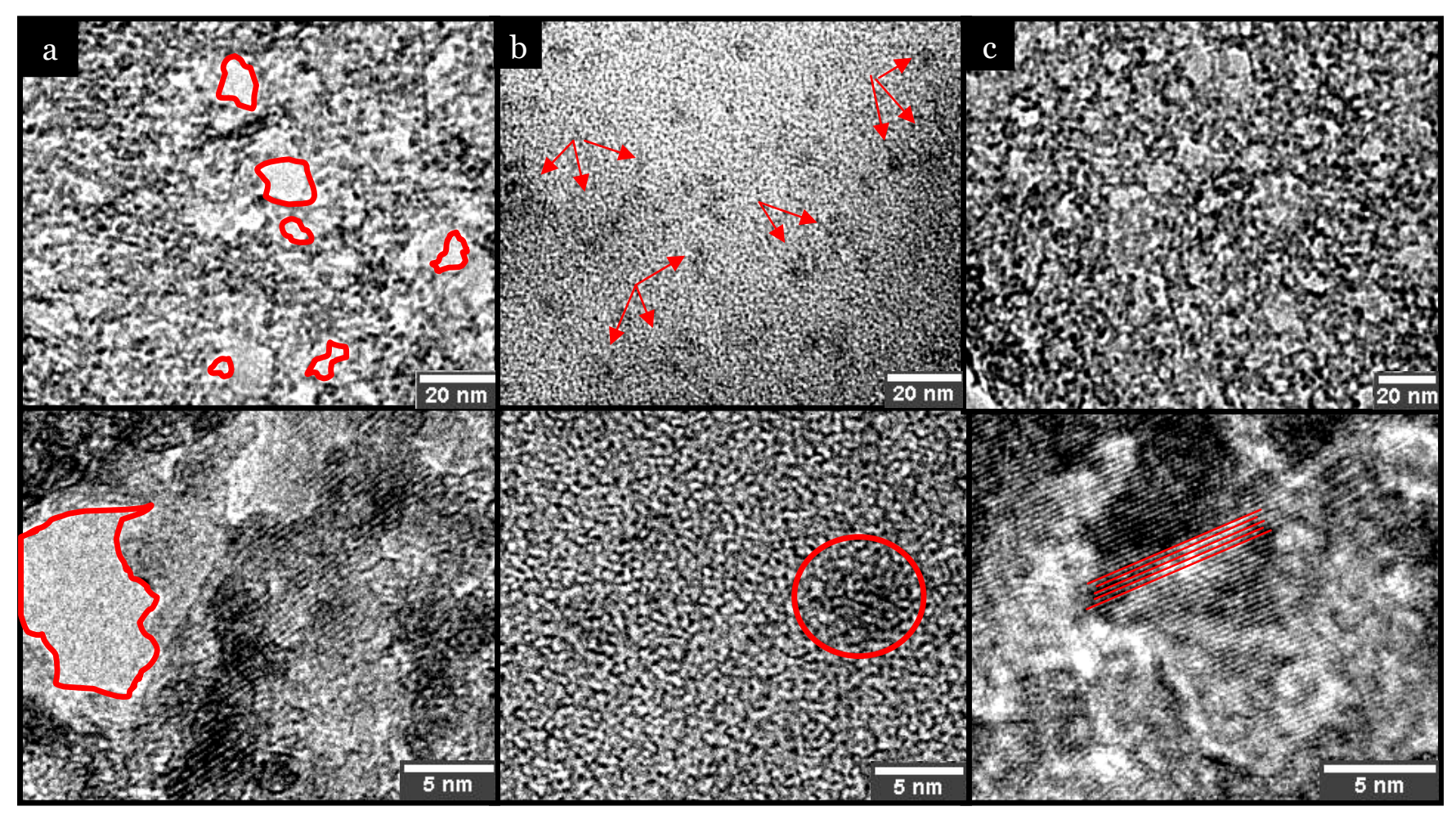

Figure 2. 


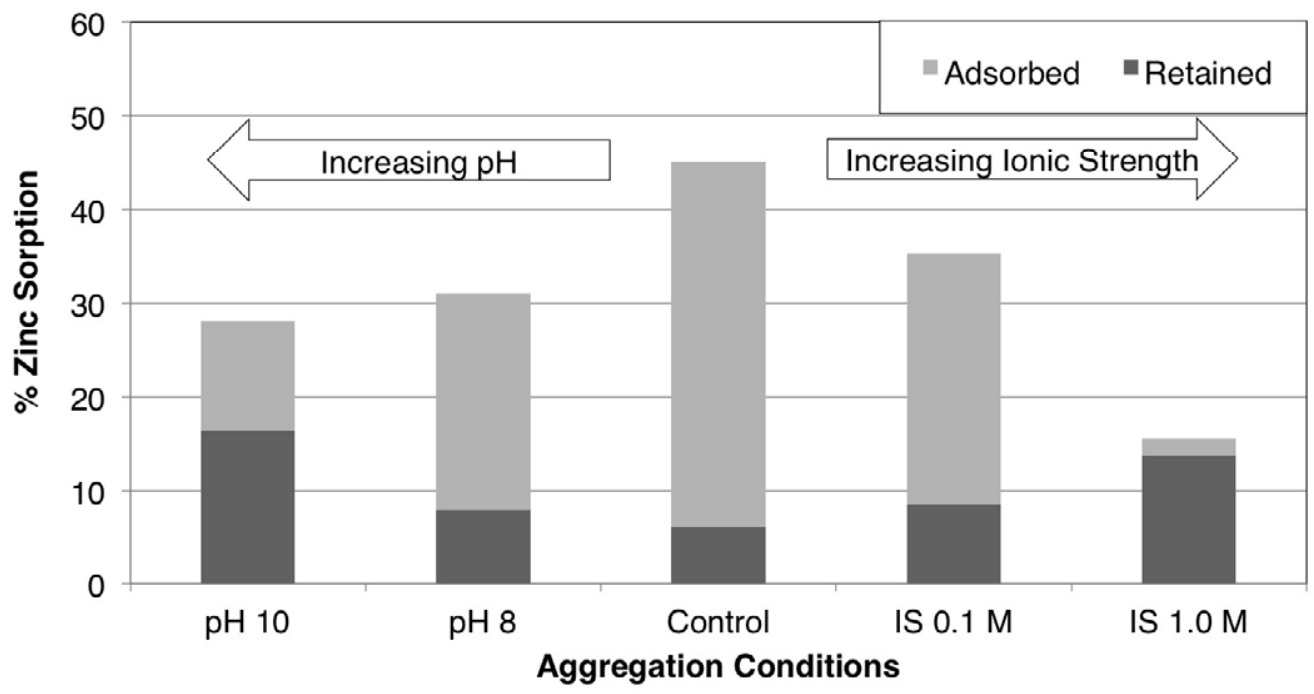

Figure 3. 

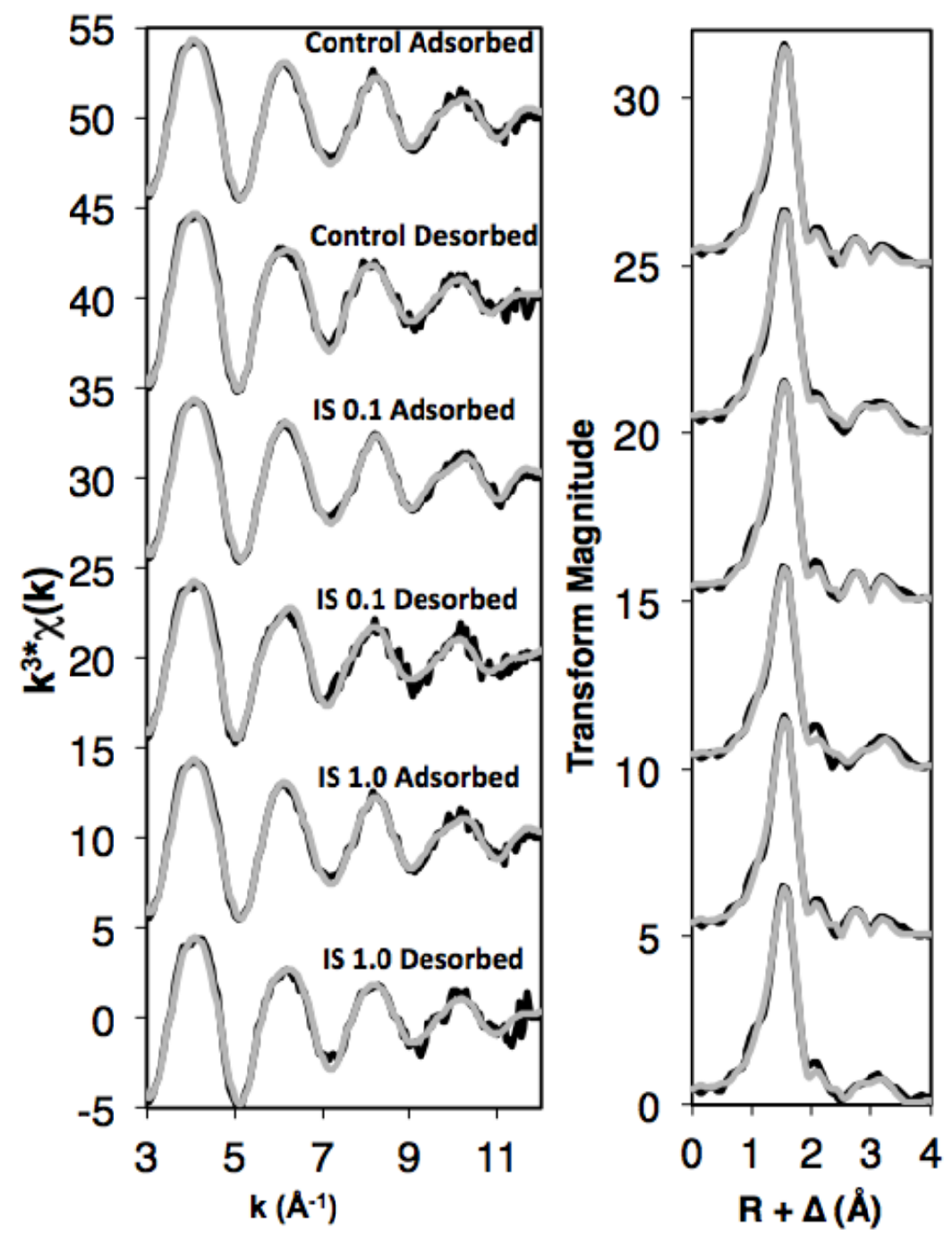

Figure 4. 

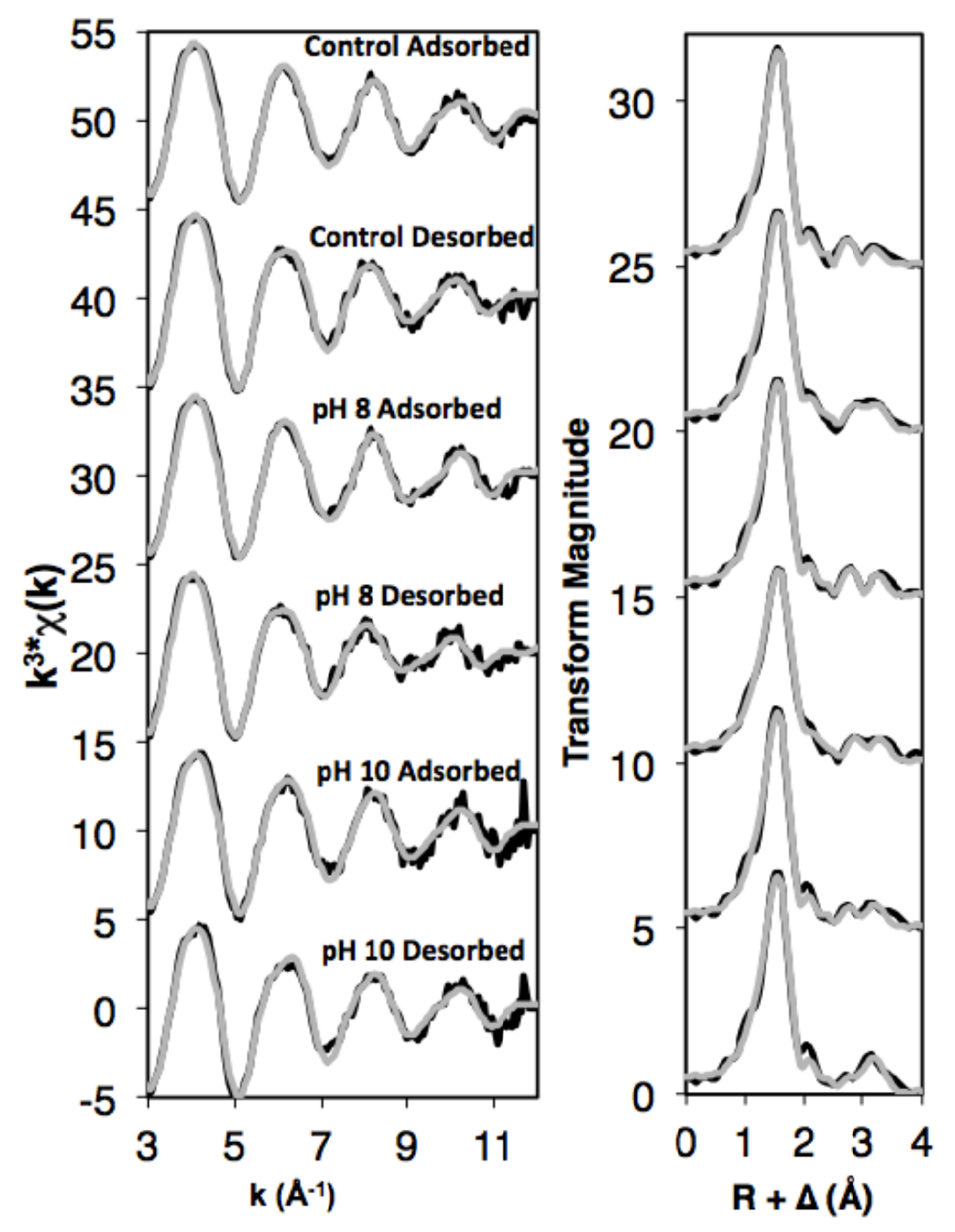

Figure 2. 

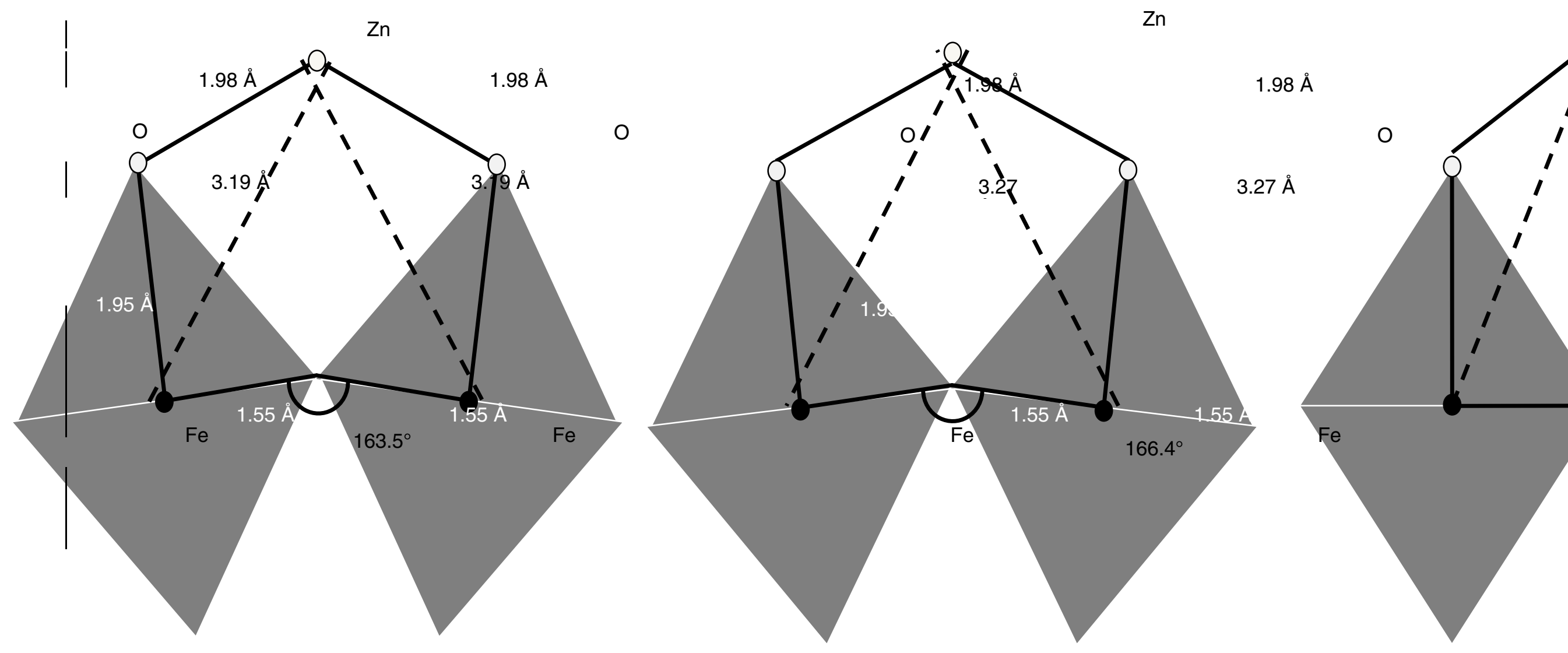

Figure 6. 


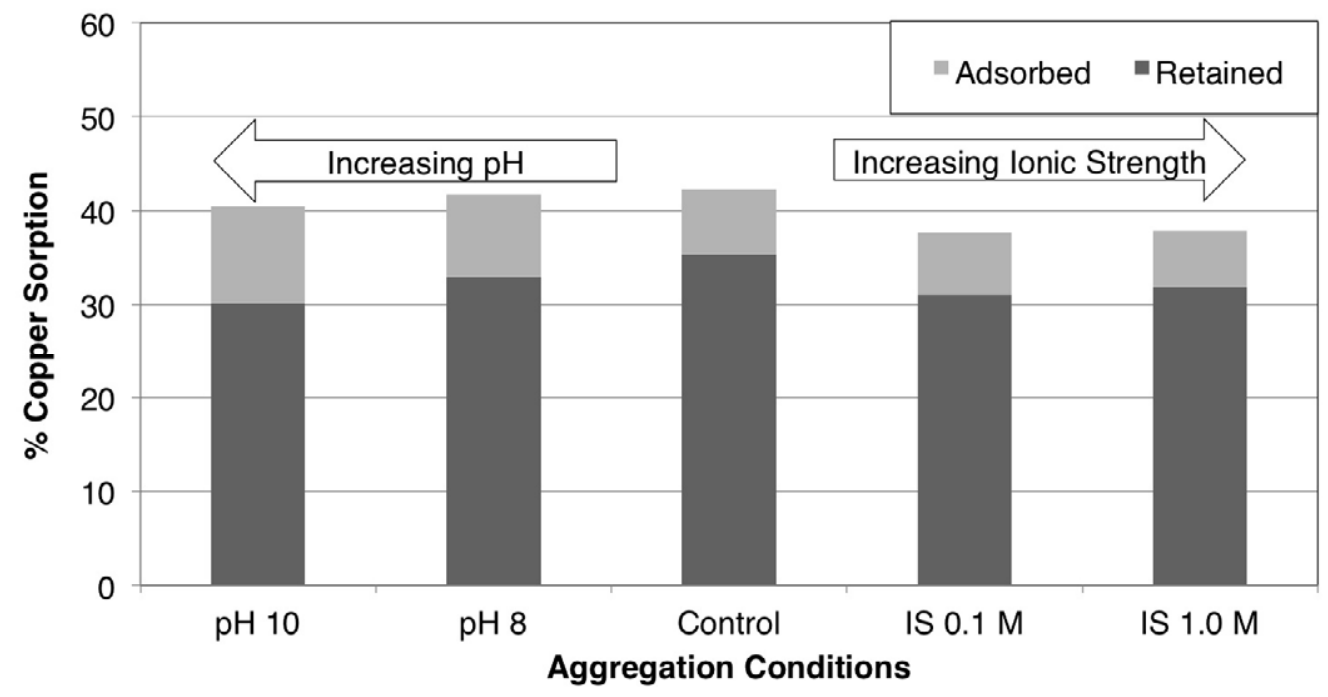

Figure 7. 


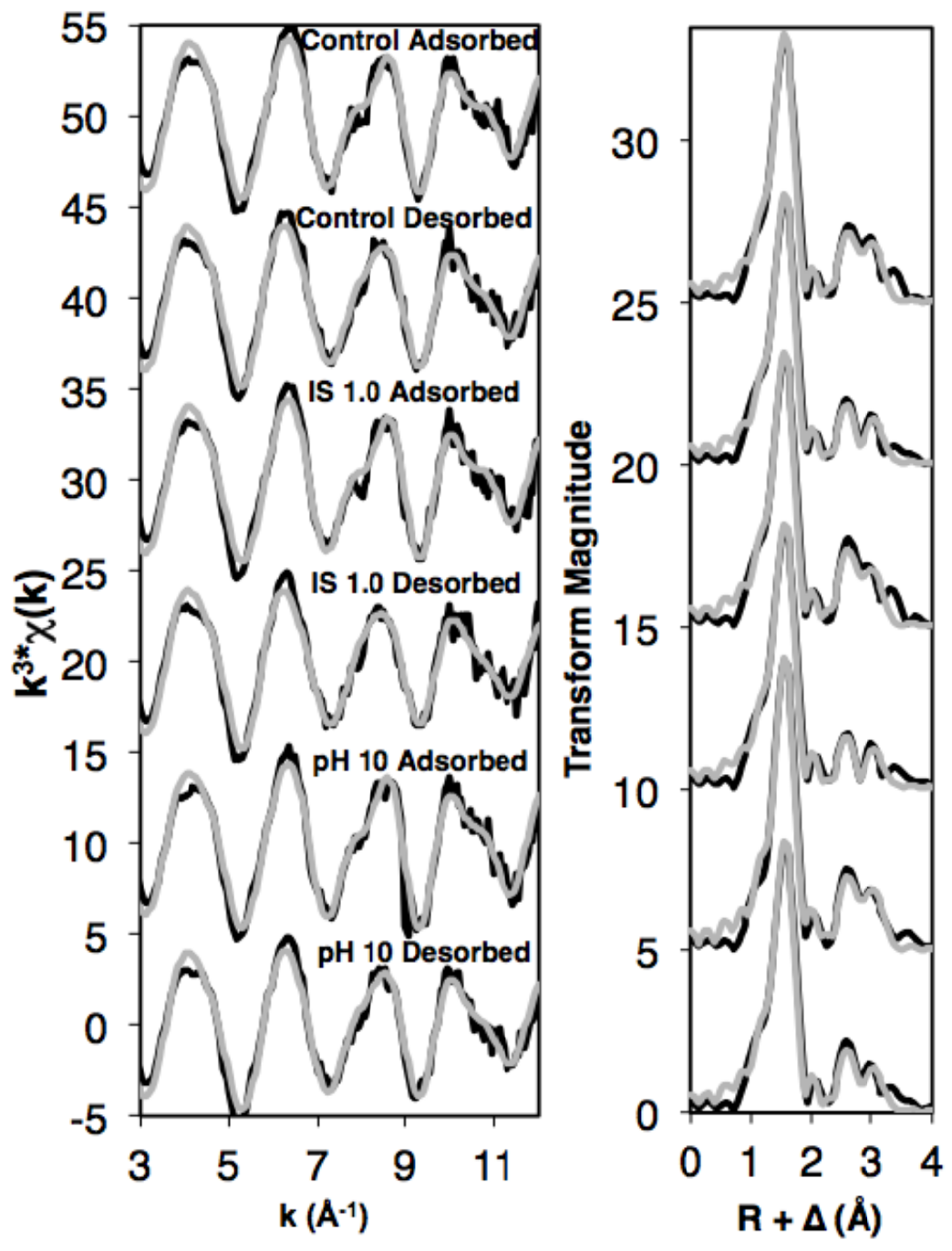

Figure 8. 